\title{
Imaging Point Defects in Complex Oxides Using Quantitative STEM
}

\author{
Honggyu Kim ${ }^{1}$, Jack Y. Zhang ${ }^{1}$ and Susanne Stemmer ${ }^{1}$ \\ 1. Materials Department, University of California, Santa Barbara, USA.
}

While methods to measure the global concentration of point defects exist, determining their spatial arrangement is significantly more challenging. Furthermore, most techniques cannot provide direct information about atom relaxations around a point defect even though these are crucial for many properties.

Previously, we have shown that HAADF-STEM can provide three-dimensional information of the location of individual dopant atoms in $\mathrm{SrTiO}_{3}$ from a single image [1]. The number of dopant atoms in a column and the depth position information are extracted using quantitative STEM, by comparing the experimental column intensities with calculations for all possible dopant configurations, and determining the most probable dopant position given an experimentally determined noise function. This method is limited by inherent experimental noise (detector noise, sample instability under the beam, sample contamination, surface amorphous layers, sample imperfections, etc.), in particular, when intensity differences between different configurations are small.

The contrast and interpretability can be improved using variable-angle HAADF-STEM (VA-HAADF). A key feature of VA-HAADF is that it utilizes information provided by the angular dependence of the scattering in HAADF (Fig. 1). This angular dependence depends on the dopant depth position. As the incident probe channels along a column, atoms deeper in the foil see a more focused probe, resulting in scattering to higher angles, relative to a dopant atom nearer to the entrance surface. Thus, by selecting certain angular ranges, different dopant atom configurations may be more easily distinguished. VAHAADF allows for precise and accurate determination of three-dimensional dopant atom configurations in doped $\mathrm{SrTiO}_{3}$ films [2]. The acquisition of multiple variable angle data and use of compound probabilities significantly improved the accuracy and precision of both the number of dopants as well as the their position(s). Configurations that are ambiguous in one detector regime can be resolved in the other.

Detecting vacancies in STEM images is even more challenging than dopant atoms that have a large atomic number difference with the host. We have combined VA-HAADF and rigid registration methods to detect $\mathrm{Sr}$ vacancies in $\mathrm{SrTiO}_{3}$ and their associated local atom relaxations [3]. Lattice relaxation around the vacancies are detected with picometer precision (Fig. 2). Rigid registration methods not only improve the precision in measurements of atom column positions but also the quantification of image intensities, which allows for the detection of point defects with low Z-contrast, such as vacancies. The number of vacancies in each column can be quantified. Discrepancies between the measured lattice relaxations and predictions from DFT simulations underscore the need for atomic-scale experimental input in developing a microscopic understanding of how point defects control real materials properties [4]. 


\section{References:}

[1] J. Hwang et al, Phys. Rev. Lett. 111 (2013) 266101.

[2] J. Y. Zhang et al, Sci. Rep. 5 (2015) 12419.

[3] H. Kim et al, Phys. Rev. X 6 (2016) 041063.

[4] The authors acknowledge support by the U.S. Department of Energy (Grant No. DEFG0202ER45994).

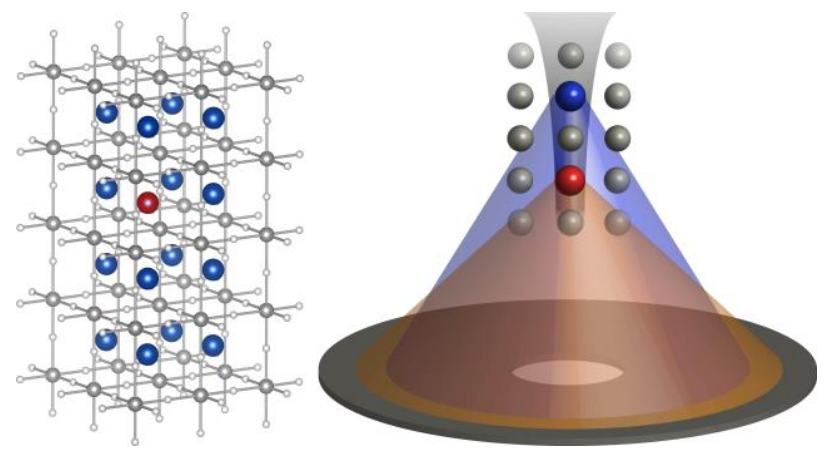

Figure 1. VA-HAADF, which utilizes different angular ranges of the detector. One particular detector range is not unilaterally better than the other but using multiple angle ranges and compound probabilities significantly improve depth quantification.

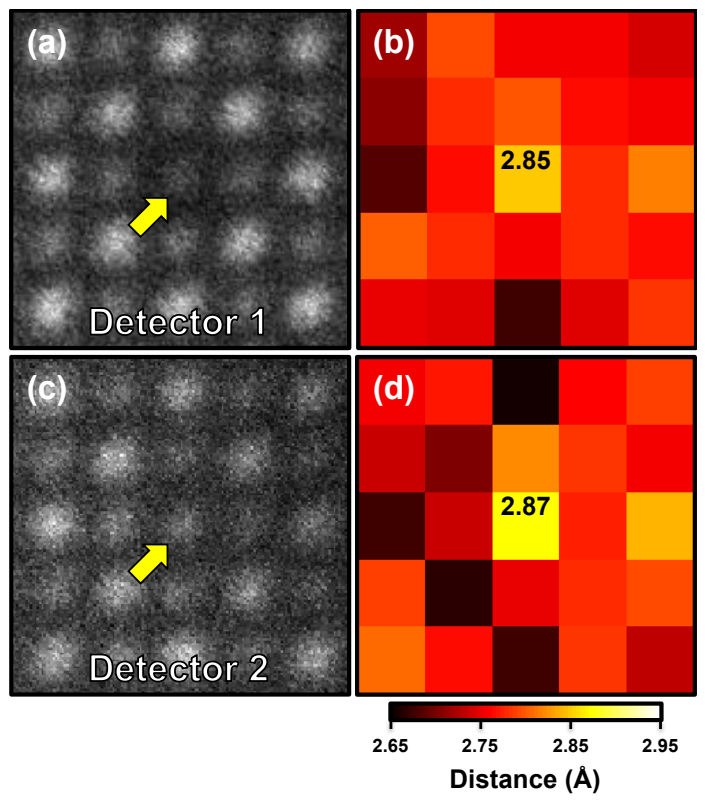

Figure 2. Lattice relaxations around a column containing $\mathrm{Sr}$ vacancies in $\mathrm{SrTiO}_{3}$ (see arrows). The distance maps, shown on the right, indicate the averaged distance from the column to its four nearest neighbor columns and show that the Ti-O columns are displaced away from $\mathrm{Sr}$ vacancies. 